\title{
Piercing Quasi-Rectangles: On a Problem of Danzer and Rogers
}

\author{
János $\operatorname{Pach}^{1, \star}$ and Gábor Tardos ${ }^{2, \star \star}$ \\ 1 EPFL, Lausanne and Rényi Institute, Budapest \\ pachecims . nyu . edu \\ 2 Department of Computer Science, Simon Fraser University, \\ Burnaby and Rényi Institute, Budapest \\ tardos@cs.sfu.edu
}

\begin{abstract}
It is an old problem of Danzer and Rogers to decide whether it is possible arrange $O\left(\frac{1}{\varepsilon}\right)$ points in the unit square so that every rectangle of area $\varepsilon$ contains at least one of them. We show that the answer to this question is in the negative if we slightly relax the notion of rectangles, as follows. Let $\delta$ be a fixed small positive number. A quasi-rectangle is a region swept out by a continuously moving segment $s$, with no rotation, so that throughout the motion the angle between the trajectory of the center of $s$ and its normal vector remains at most $\delta$. We show that the smallest number of points needed to pierce all quasi-rectangles of area $\varepsilon$ is $\Theta\left(\frac{1}{\varepsilon} \log \frac{1}{\varepsilon}\right)$.
\end{abstract}

* Supported by NSF Grant CCF-08-30272, by OTKA, and by Swiss National Science Foundation Grant 200021-125287/1.

** Supported by NSERC grant 329527, OTKA grants T-046234, AT-048826, and NK-62321, and by the Bernoulli Center at EPFL. 